\title{
Window technique for climate trend analysis*
}

\author{
Tamás Szentimrey, Tibor Faragó, and Sándor Szalai \\ Hungarian Meteorological Service, P.O. Box 38, H-1525 Budapest, Hungary \\ Received October 15, 1990/Accepted August 2, 1991
}

\begin{abstract}
Climatic characteristics are affected by various systematic and occasional impacts: besides the changes in the observing system (locations of the stations of the meteorological network, instruments, observing procedures), the possible local-scale and global natural and antropogenic impacts on climatic conditions should be taken into account. Apart from the predictability problems, the phenomenological analysis of the climatic variability and the determination of past persistent climatic anomalies are significant problems, among other aspects, as evidence of the possible anomalous behavior of climate or for climate impact studies. In this paper, a special technique for the identification of such "shifts" in the observational series is presented. The existence of these significant shorter or longer term changes in the mean characteristics for the properly selected adjoining periods of time is the necessary condition for the formation of any more or less unidirectional climatic trends. Actually, the window technique is based on a complete set of orthogonal functions. The sensitivity of the proposed model on its main parameters is also investigated. This method is applied for hemispheric and Hungarian data series of the mean annual surface temperature.
\end{abstract}

\section{Introduction}

The hypothesis of enhanced greenhouse gas phenomenon is apparently well reinforced by the latest observations: the last decade was the warmest one of the century and the overall temperature trend indicates global warming (Hansen and Lebedeff 1987, 1988). This means a rise in the average temperature of about $0.6^{\circ} \mathrm{C}$ as compared with the 1880 s situation (including corrections for the urban effects). Jones and Kelly (1983) pointed out that significant warming took place for the

\footnotetext{
* Contribution to Clima Locarno - Past and Present Climate Dynamics; Conference September 1990, Swiss Academy of Sciences - National Climate Program
}

Northern Hemisphere during the period 1917-1940 then it was followed by some cooling until 1965; afterwards the warming tendency continued. Angell (1988) estimated the warming rate for the Northern Hemisphere for the recent three decades as $0.08^{\circ} \mathrm{C} /$ decade $\left( \pm 0.09^{\circ} \mathrm{C}\right)$. A detailed study of the relations of the data coverage for the Northern Hemisphere and the estimated tendencies in the temperature records is given by Jones et al. (1986). Most authors revealed that a rapid warming started in the 1920 s with its maximum in the late $1930 \mathrm{~s}$, it was followed by a relatively short-term cooling tendency and now the significant warming seems to be continuing during the recent decade. The overall estimate of the positive trend for the centuryscale warming is about 0.0 to $0.6^{\circ} \mathrm{C}$ for the Northern Hemisphere according to different research groups. It should be noted that strong criticism about these results is also raised by some authors especially because of the inconsistencies in the observations used for the climatic change detection studies (Callendar 1961; Karl and Quayle 1988). The trend detection in the past and recent observational series on smaller spatial scales is also important for the estimation of the predictability value of climatic normals or any other climatic characteristics (Dixon and Shulman 1984).

Large-scale average surface temperature observations are used in many studies as indicators of the possible climatic change. The problems of derivation of these data series have been dealt recently by several research groups; an overview is given by Wigley et al. (1986). Long-term temperature records for the Northern Hemisphere (mainly based on continental observations) were derived and reported, inter alia, by Jones and Kelly (1983), Jones et al. (1986), Vinnikov and Groisman (1981), Vinnikov et al. (1987), Hansen and Lebedeff $(1987,1988)$. The interpretations of these records by the authors are rather similar as regards the likelihood of a warming trend with the slight differences in the phase and amplitude estimates.

The relation between the global and local tendencies is even more difficult ( $\mathrm{Kim}$ et al. 1984). As a matter of fact, no single station can be found which temperature 
variations (or climate, in general) is in significant correlation with the large-scale average value. It means that the area-averaging is a principal attribute of the global scale results. This aspect is obvious from a statistical point of view, however, its proper consideration is sometimes neglected. Regional temperature trends were studied, among others by Agee (1982), Williams and van Loon (1976), Karl and Quayle (1988). It should be emphasized that the behavior of the smaller scale noise terms being inherent in the local or regional observations play a determining role in the analysis of their "coherence" with the global (globally averaged) sample series.

Various methods were used in the trend analysis of the records. The independence of the sample series elements is accepted in the most studies with fitting of the ordinary linear or non-linear regression model (Angell 1983; Jones and Kelly 1983). More sophisticated approaches are also proposed, like the two-phase (piecewise linear) estimate by Solow (1987). Madden and Ramanathan (1980), Wigley and Jones (1982) assumed that the noise term follows a first-order autoregressive scheme. Significance tests of the results are performed, for instance, by the t-test (Karl and Riebsame 1984; Gruza et al. 1987). Apparently, beside such characteristics of the sample series as the size of the sample, the spatial resolution of the observations, the method of their averaging, the combination of the local and global impacts on the observations, the results on the climatic trends depend on the particular models to large extent. The suggested functional forms are sometimes oversimplified. Instead, the climatic trend should be interpreted in a very extended sense: it can be of arbitrary (parametric or non-parametric) functional form, which means the use of the general concept of climatic signal and noise. Also, the interpretation of the observations, the identification of the trends depends to a very large extent on the period or time window for which the time series characteristics are calculated. Some of these problems are addressed in details in the literature (e.g., Jones and Kelly 1983; Jones et al. 1986; Karl and Quayle 1988).

In our paper, we focus on those methodological problems of trend detection which are related to the identification of significant anomalies (anomalous subperiods) in the observational data series and which substantially depend on the concrete time period of observations used for particular trend detection studies. Illustrations are given simultaneously for average annual temperature data for the Northern Hemisphere and Hungary. It is believed that such time series analysis and detection studies should be performed for global and regional data as well, however, only the former data can serve as indicators (or primary indicators) of the possible global climatic change. However, we keep in mind that "the reliance on the annual Northern Hemisphere average in many studies of recent climatic change is unfortunate, reflecting more, we believe, its convenience than its value as a physical parameter" (Jones and Kelly 1983). In addition, in both cases, the phenomenological analysis of past data is important for various purposes: for instance, for an explanatory part of the climate impact case studies of contemporary climate-related events or to provide evidence for recent anomalous behavior of the climate (Epstein 1982). In our numerical experiments, the emphasis is made primarily on the methodological aspects of the time series analysis and the demonstration of the proposed method.

\section{The trend models and the window technique}

The trend models are set under the assumption that the time series consists of two parts, namely

$\delta_{t}=m_{t}+\varepsilon_{t} \quad t=1,2, \ldots, n$

where $m_{t}$ is the trend function, i.e., changes of the variable $\delta_{t}$ can be characterized by this trend function $m_{t}$, and $\varepsilon_{t}$ is the white noise term with zero mean (expected value) and variance $\sigma^{2}$. In this case, the variance $\sigma^{2}$ is the measure of variability for the investigated variable.

The purpose of the statistical trend analysis is to separate the trend and the noise terms. For the evaluation of the given model, a set of hypotheses about the validity of the trend attributes should be tested by means of statistics at a given significance level. We wish to get as much significant information as possible about the trend and this requirement is essentially equivalent to the total (mutual) independence of statistics. In this respect, the application of the orthogonal series expansions is advantageous in the case of normal noise.

These general conditions can be satisfied by several approaches. The linear analytical trend analysis is the most commonly used scheme, its time-dependent term can be tested and interpreted physically very well, but it gives only a particular and sometimes even misleading information about the general trend function. There is no reason to assume that even if the general warming tendency is accepted, its rate obeys a unique simple functional form. Discrete Fourier-series expansion or periodogram analysis are other trend approximations which produce independent statistics, however, their

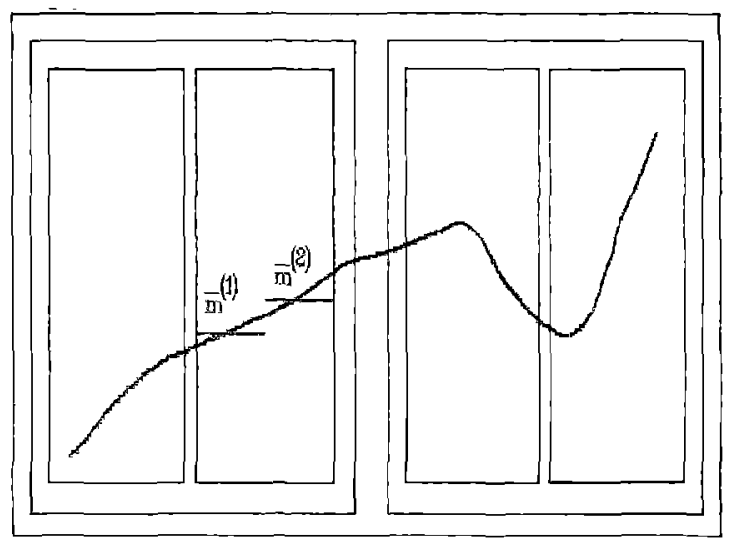

Fig. 1. General concept of the window technique for trend analysis: the most significant "breaks" of the observational series are found by use of subsequent windows or subperiods and the evaluation of local mean values for these subperiods 
"physical" interpretation raise certain principal problems.

Taking into consideration these shortcomings, we suggest the application of a special window technique which enables us to reveal the significant changes in the investigated time series and requires minimum assumptions about the parameterizable functional form of the trend to be present (or not present) in the climatic time series.

The window technique is based on the Haar-series expansion (Riesz and Sz.-Nagy 1953; Kolmogorov and Fomin 1960). The specific purpose of this trend analysis is to get information about the changes of the mean characteristics of the observational series within certain time intervals. More exactly, the (statistical) decision about the acceptability of hypothesis $\bar{m}_{(1)}=\bar{m}_{(2)}$ should be tested for the elements of a given window (sub-period) system, where $\bar{m}_{(1)}, \bar{m}_{(2)}$ are the corresponding averages of the trend function (Fig. 1).

\section{The Haar-function system}

The essence of the Haar-series expansion is the following. Let $I$ be a sub-interval system of $[0,1]$. By definition $[0,1] \in I$ and if a closed interval belongs to the system, $[k, l] \in I$ then $[k,(k+l) / 2] \in I$ and $[(k+l) / 2, l] \in I$, that is both halves of the particular intervals are also the elements of the system. In this case, the step-wise functions $\left\{\Phi_{[k, l]}(t) ;[k, l] \in I\right\}$

$\Phi_{[k, l]}(t)=\left\{\begin{array}{cll}-1 / 2 & \text { if } & t \in[k,(k+l) / 2) \\ 1 / 2 & \text { if } & t \in((k+l) / 2, l] \\ 0 & \text { if } & t \in([0,1] \backslash k, l]) \vee\{(k+l) / 2\}\end{array}\right.$

together with the function $\Phi^{0}(t) \equiv 1, t \in[0,1]$ form a complete orthogonal system within the function space $L^{2}([0,1])$. It means that all members of this function space can be expressed by means of this system as the sum of its projections on the elements of the orthogonal system.

\section{The window functions}

The concept of the Haar-function system can be extended to arbitrary intervals and its discrete subsets. Let us introduce the following discrete (time) interval system $I$

$$
\begin{aligned}
& (1, n)=\{1,2, \ldots, n\} \in I \\
& \text { if }(k, l)=\{k, k+1, \ldots, l] \in I, k<l \text { then } \\
& \quad(k,[(k+l) / 2)=\{k, \ldots,[(k+l) / 2]\} \in I \text { and } \\
& \quad([(k+l) / 2]+1, l)=\{[(k+l) / 2]+1, \ldots, l\} \in I
\end{aligned}
$$

where $[(k+l) / 2]$ denotes the integer part of $(k+l) / 2$.

Then the windows are defined as the discrete intervals which have at least two elements, that is:

$W=\{(k, l) \mid w \geq 2,(k, l) \in I\}$,

where $w=l-k+1$ is the width of the interval $(k, l)$. In this case the number of windows is \#W=n-1.

The generalized Haar-functions $\left\{\Phi_{(k, l)}(t) ;(k, l) \in W\right\}$ are defined upon this window system, namely $\Phi^{0}(t) \equiv 1$ and

$$
\begin{aligned}
& \Phi_{(k, l)}(t)=-[w / 2] / w \quad \text { if } \quad t \in(k,[(k+l) / 2]) \\
& {[w / 2] / w \text { if } t \in([(k+l) / 2]+1, l) .}
\end{aligned}
$$

where $w$ is the width of the window $(k, l)$, and [w/2] denotes the integer part of $w / 2$. These functions form a complete orthogonal step function system upon the set of (ordered) values $\{1, n\}$ analogously to the Haar-function system in the discrete case.

The expansion of the time series of investigated climatic observations and their trend upon the above function system can be written in the form:

$$
\begin{gathered}
m_{t}=\bar{m}_{(1, n)}+\Sigma d \bar{m}_{(k, l)} \Phi_{(k, l)}(t), \quad(k, l) \in W \\
\delta_{t}=\bar{\delta}_{(1, n)}+\Sigma d \bar{\delta}_{(k, l)} \Phi_{(k, l)}(t),(k, l) \in W,
\end{gathered}
$$

with the coefficients of these expansions being just the values of the changes in the time averages:

$d \overline{\boldsymbol{m}}_{(k, l)}=\overline{\boldsymbol{m}}_{([(k+l) / 2]+1, l)}-\overline{\boldsymbol{m}}_{(k,[(k+l) / 2])}$ $d \bar{\delta}_{(k, l)}=\bar{\delta}_{([(k+l) / 2]+1, l)}-\bar{\delta}_{(k,[(k+l) / 2])}$,

where $\bar{m}_{(i, j)}, \bar{\delta}_{(i, j)}$ denote the averages for interval $(i, j)$.

\section{Test of hypothesis on the particular climate change episodes}

The main objective of the extended Haar-series expansion is the selection of coefficients $d \bar{m}_{(k, l)}$ which differ from zero on the basis of estimators $d \bar{\delta}_{(k, l)}$. In other words, those particular episodes are looked for where significant changes in the successive subperiod means are present. We emphasize already at this point that the identification of such particular changes does not mean a jumplike change in the climate. Such climatic jumps were studied by Knox et al. (1988). The principal idea behind our findings is that most changes (of usually unknown shape and rate) also lead to the changes in the local mean values for adequately chosen successive subperiods of time. Therefore, such changes in the means can be considered as indicators of climatic change, however they do not give, but rather only approximate, the specific form of the actual climatic signal.

As a consequence of using orthogonal series expansion and assumption on normality, the estimators $d \bar{\delta}_{(k, l)},(k, l) \in W$ are totally independent and normally distributed, $d \bar{\delta}_{(k, l)} \in N\left\{d \bar{m}_{(k, l)} ; \quad w^{*} \sigma^{2} /([w / 2](w-[w /\right.$ 2]))\}.

On the basis of these features, statistical procedure can be constructed for the detection of non-zero differences in the successive climatic means $d \bar{m}_{(k, l)} \neq 0$ at a given significance level. For this purpose, the ordered squares of standardized estimators $d \bar{\delta}_{(k, l)}$ $(w=l-k+1>3)$ are examined, while the estimators $d \bar{\delta}_{(k, l)}(w=l-k+1 \leq 3)$ are applied to estimate the unknown "noise" variance $\sigma^{2}$. The latter procedure is adequate if it is assumed that $\left(d \bar{m}_{(k, l)}\right)^{2} \ll \sigma^{2}$ for $w=l-k+1=2,3$ i.e., the rate of change compared with ("interannual") climatic variability is slow. The critical values of this hypothesis test which correspond to the 
given significance level can be calculated by MonteCarlo simulations.

According to this statistical procedure, if $K$ smaller or larger but jointly significant "changes" are accepted:

$d \bar{m}_{(k, l)}^{(1)} \neq 0, \ldots, d \bar{m}_{(k, l)}^{(K)} \neq 0$,

then the probability of first type error (the validity of the existence of a coefficient $\left.d \overline{\boldsymbol{m}}_{(k, l)}^{(i)}=0(i=1, \ldots, K)\right)$ is less than or equal to $p$, where $1-p$ is the given significance level.

One of the most advantageous features of the window technique is that the trend can be approximately reconstructed as the combination of estimates of the particular changes. Taking into account only those components which were accepted to be significant, the trend estimate is simply the "truncated" expansion of the time series in Eq. (3):

$\hat{m}_{t}=\bar{\delta}_{(1, n)}+\Sigma d \bar{\delta}_{\left(k_{2}, l\right)}^{(i)} \Phi_{(k, l)}^{(i)}(t), i=1, \ldots, K$,

with those indices for which $d \bar{m}_{(k, l)}^{(i)} \neq 0(i=1, \ldots, K)$ at the given significance level.

\section{Applications}

\section{The data series}

Two sample series are studied simultaneously. The annual mean surface air temperature record for the period 1851-1988 for the Northern Hemisphere (Jones et al. 1986; Jones et al. 1990) was used for the analysis of the global trend detection problems. This series will be referred to as $\mathrm{N}$-series with the indication of the period if necessary. This data set was used on the one hand, to reveal long-term changes in the average surface temperature, on the other hand, its reduced version (since 1881) served as a parallel data basis for our regional data.

The annual mean temperature record for Hungary has been derived as the spatial average of selected Hungarian stations for the period 1881-1988 (denoted further as $\mathrm{H}$-series). For this purpose, observations of 16 meteorological stations with long climatic records were involved, however, no special homogeneity or other tentative data screening analysis was applied. These stations cover the whole area of the country. We admit that observations at some of the selected stations can be influenced by gradually strengthening urban effects (and other factors) so that we interpret the results of the present trend analysis with proper care. Similar problems arise for the larger-scale data which is the source of uncertainties and speculations about the observational evidence of the climatic change (Karl and Quayle 1988).

\section{Application of window method to temperature series}

As mentioned, various temperature series and methods are used for trend estimates on global and hemispheric scales. The most common technique because of its ultimate simplicity is the linear trend model which gives a firm positive trend for the N-series (Fig. 2). It is usually interpreted as an indication of global warming and expressed in terms of the warming rate which is the mean temperature rise per decade. We think that the available observations or the physical background (buildup of greenhouse gases in the atmosphere) do not give enough basis to make conclusions on the functional form of this tendency in the temperature series. The other methodologically possible alternative is the use of non-parametric estimators (moving weighted averages, splines). However, such models are of rather limited value because usually neither the problem of detection of the change in the data series is solved, nor do they have at least formal predictive capabilities. To deal with the former problem, obviously, one can avoid attaching a parameterizable specific analytical form to the observations. If a more or less monotonous climatic signal (with unknown form and rate of change) is present in the observational series, the time averages will follow the tendency of the signal. The window technique is just the generalization of this simple concept. Applying this method to the $\mathrm{N}\{1851-1988\}$-series, only a single significant change can be detected at significance level 0.9 (Fig. 2)

\section{$\bar{m}(1851,1919)<\bar{m}(1920,1988) \quad$ (138-year window).}

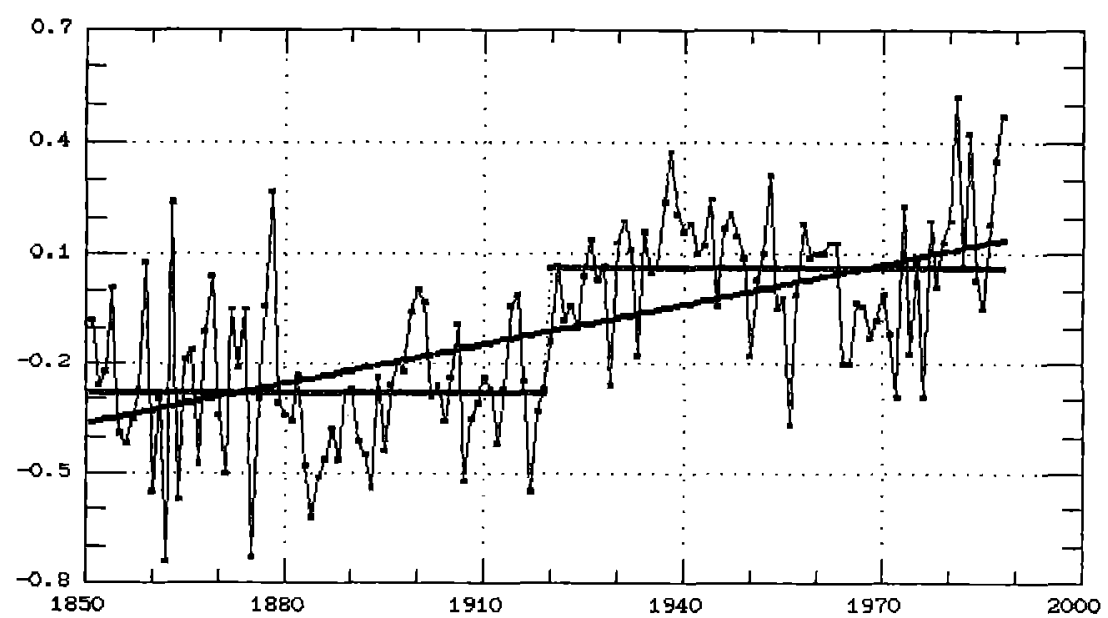

Fig. 2. The linear trend and the trend estimate by the significant elements (at level 0.9 ) of the window function system for the hemispheric temperature record for period 1851-1988 
This result only means that a statistically significant positive increase in the Northern Hemispheric surface temperature series could be detected. No further refinement of the trend is justifiable in this window model at the same significance level, though temperature increase during both halves of the whole period could be expected according to the linear approximation.

Beside the effects of the prescribed functional forms or models of trend, the use of observations for concrete time periods determines the identification and determination of the underlying trend component. In other words, the allocation and size of the window (sample from a subperiod) with respect to the available observational series can be a determining factor.

Given a particular observational series, say the Nseries, it is worth performing a retrospective analysis by reducing the series or taking various initial windows. This way, we cut the $\mathrm{N}$-series first by selecting the period 1881-1988 (Fig. 3). This analysis is also needed for comparisons with the regional data (the $\mathrm{H}$-series). The significant components (at level 0.9 ) revealed are as follows:

$\bar{m}(1881,1934)<\bar{m}(1935,1988) \quad$ (108-year window)

$\bar{m}(1881,1907)<\bar{m}(1908,1934) \quad$ (54-year window)

$\bar{m}(1908,1921)<\bar{m}(1922,1934) \quad$ (27-year window)

$\bar{m}(1962,1975)<\bar{m}(1976,1988) \quad$ (27-year window)
Therefore, the window technique allows us to have much more significant features for this reduced period: on the one hand, the linear estimate seems to be even less adequate for this case, on the other hand, two wellknown features of the temperature course are also identified as significant changes in the subperiod mean values. Namely, the relatively warm $30-40$ y period following the early 1920 s and the peculiar temperature drop during the 1960 s and early 1970s. Parallel with these calculations, the Hungarian data for the same period 1881-1988 were studied (Fig. 4). Its linear trend has a near zero slope, whereas its Haar-analysis results in a single significant component (at 0.9 level), namely:

$\bar{m}(1935,1961)>\bar{m}(1962,1988) \quad$ (54-year window)

and for all other windows the hypotheses on changes at the local means are rejected at significance level 0.9 . Obviously, the warming period after the late 1920 s coincides with the larger-scale tendencies, however no significant warming could be identified from these data after the temperature decrease which started in the 1960s. The unidentified significant warming subperiod during the 1980s does not mean a conceptual contradiction to the hemispheric temperature trend which represents a large-scale spatial average. Moreover, as mentioned before, this regional observational data should be further analyzed for its representativeness.
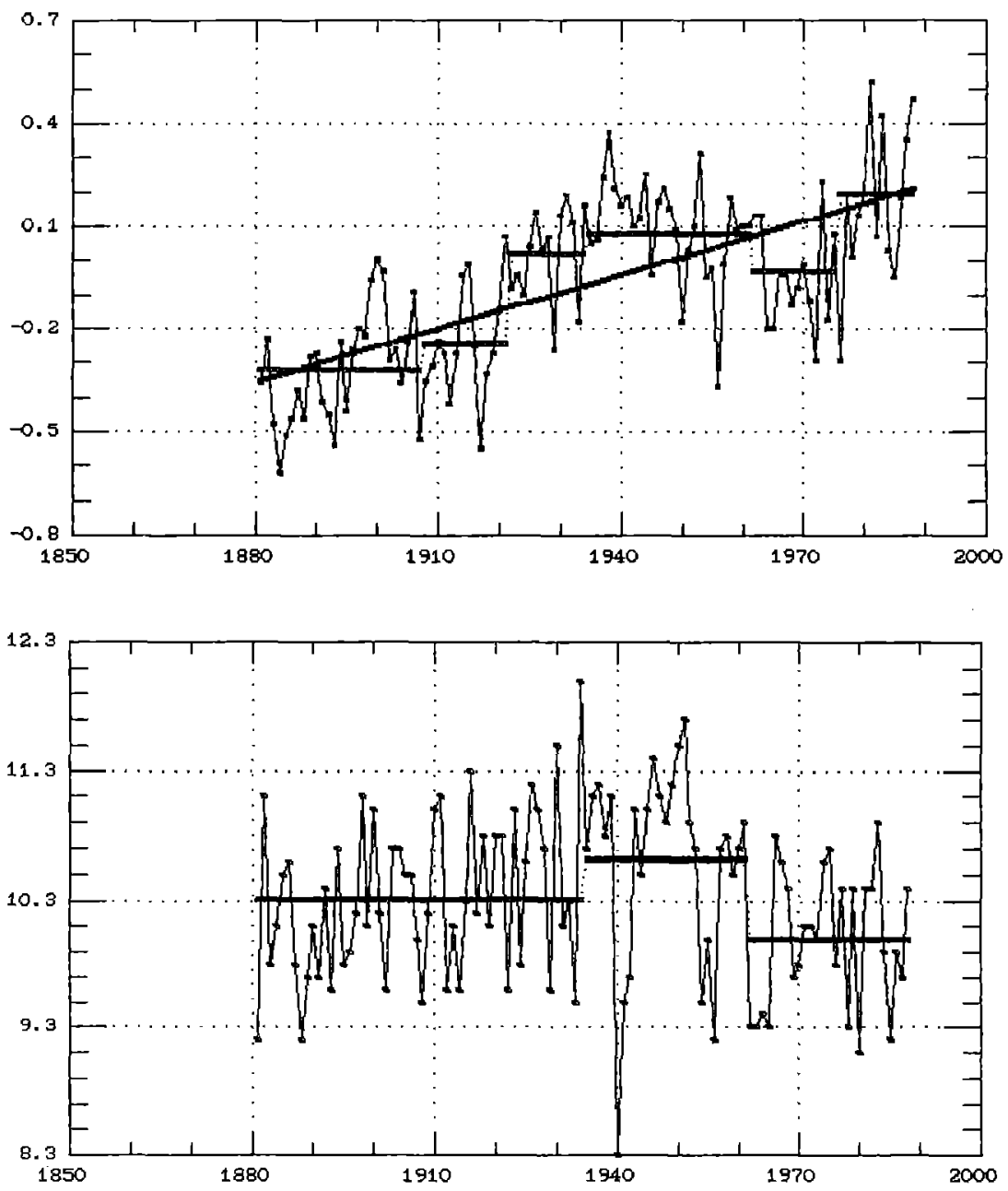

Fig. 3. The linear trend and the trend estimate by the significant elements (at level 0.9 ) of the window function system for the hemispheric temperature record for period 1881-1988
Fig. 4. The trend estimate by the significant elements (at level 0.9 ) of the window function system for the Hungarian temperature observations for period 1881-1988 
In another numerical experiment, we go "back" to the middle of the 1970s (Fig. 5). The window approximation for the hemispheric data $\mathrm{N}$ (1881-1977\} shows the same general shape as that for the larger window $\mathrm{N}$ [1881-1988\}, however, its right hand side represents the significant drop in the average surface temperature. That time, this phenomenon gave a basis for speculations about the beginning of global cooling. It is a good indication for the sensitivity of such commonly used terms as global cooling or warming. After a decade of couple of record-breaking temperatures, there is again a "general consensus" about the global warming trend. Instead, we suggest interpretation of the observational series and the trends fitted to them with proper care.

\section{Sensitivity of the trend estimates}

We should note that the various trend models are more or less sensitive to the data series. The sensitivity is commonly investigated in terms of the model's stability to changes (or errors) in the observations. One of the straightforward properties of the simple analytical approaches (with parameterizable analytical trend functions like linear or quadratic trend functions) is that they are rather stable even to individual "outliers" (large deviations from the general trend line) in the case of relatively large sample sizes. This is also valid for the gradual extension or reduction of the investigated period.
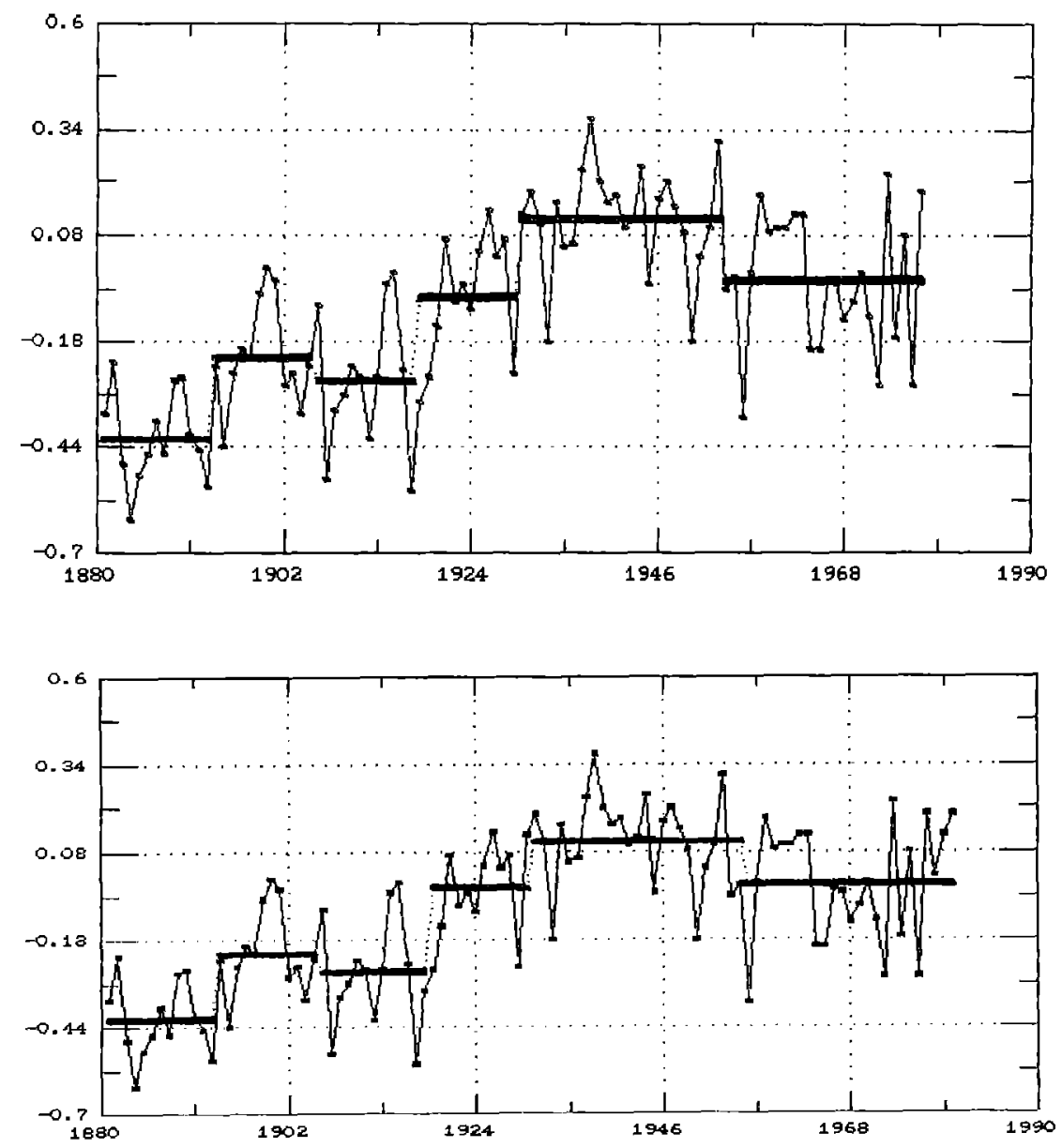

Fig. 5. The trend estimate by the significant elements (at level 0.9 ) of the window function system for the hemispheric data for period 1881-1977
Fig. 6. Sensitivity analysis of the window technique: trend estimates for the hemispheric data for the periods 1881-1980 and 1881-1982, respectively (at level 0.9 ) 
Contrary to analytical models, the window approximation is much more sensitive to changes in the observational period. The reason is a consequence of the specific structure of the windows and window functions. In particular, the extension of the initial series with a pair of new observations $n^{\prime}=n+2$ will change both subsets for the test of the first window component from $(1,[(n+1) / 2])$ and $([(n+1) / 2]+1, n)$ to $(1,[(n+1) /$ $2]+1)$ and $([(n+1) / 2]+2, n+2)$, respectively. It means that the second subset of size $[(n+2) / 2]$ changes with three elements and if there was an even finer significant division of this subset, its right half will change again with three elements after the extension of the observational series. Obviously, the results of the test statistics can change drastically for the finer subdivisions (higher-order window components) especially in the case if the new observations represent large anomalies in terms of the subperiod mean value.

To illustrate this phenomenon, the $\mathrm{N}\{1881-1980\}$ and $\mathrm{N}\{1881-1982\}$ observational series are contrasted (Fig. 6). The window analysis for the former gives no significant change for the last 25 -year period whereas the extension of the data to 1982 (including the record breaking value for 1981) yields considerably different right hand side of the trend estimate.

\section{Comparison with linear trends}

As mentioned the probability of first type error for the decision about the non-zero coefficients of window approximation (4) is less than or equal to $p$ where $1-p$ is the given significance level. Another principal question about the goodness-of-fit of this estimate concerns the second type error, that is whether the approximation in Eq. (5) is adequate to characterize the trend function or some essential information is neglected.

In this respect, the suggested method can be compared with the most commonly applied linear trend model. We test if a significant linear component can be found in the observational series together with the window components.

The linear trend model gives a firm positive trend for the $\mathrm{N}$-series (Fig. 2, 3), namely the $t$-values and the levels of significance for the coefficient of the linear function of time are the following

$\begin{array}{lrl}\text { Data series } & t \text {-value } & 1-p \text { level } \\ \mathrm{N}\{1851-1988\} & 8.2832 & 1 \\ \mathrm{~N}\{1881-1988\} & 8.7606 & 1 \\ \mathrm{H}\{1881-1988\} & -0.2875 & 0.2257 .\end{array}$

In order to test the adequacy of the window model, it is combined with a linear term

$m_{t}=c_{0}+\Sigma c_{i} \Phi_{(k, l)}^{(i)}(t)+c_{K+1} t, \quad i=1, \ldots, K$

where $\Phi_{(k, l)}^{(i)}(t)(i=1, \ldots, K)$ are the window functions according to Eq. (5).

The hypothesis $H_{0}=\left\{c_{K+1}=0\right\}$ should be tested. It would mean that the model (5) is more adequate than linear one for the given data series. The $F$-values and levels of significance for the linear term (in an ordinary multilinear regression scheme) are derived for this test procedure

$\begin{array}{lll}\text { Data series } & F \text {-value } & 1-p \text { level } \\ \mathrm{N}\{1851-1988\} & 0.0026 & 0.0409 \\ \mathrm{~N}\{1881-1988\} & 0.2450 & 0.3783 \\ \mathrm{H}\{1881-1988\} & 0.6632 & 0.5827\end{array}$

It should be noted that the $F$-value obeys the $F$-distribution for large $n$ and little $K$ (provided the $H_{0}$ is true) independently from the selection of the window functions in the regression scheme. Consequently, the above hypothesis $H_{0}$ can be accepted for the examined time series and it means that the window technique leads to trend estimates which are at least as informative as the common linear approximation.

\section{Conclusion}

The analysis of particular temperature series by use of the window technique revealed the basic characteristics which are more or less known from other sources. However, we suggest that it is better to avoid the expression of the trends in such simple terms as the annual or decadal rate of warming or cooling for the whole period. In order to verify the general tendencies, alternative global and hemispheric data series will also be used, and more substantial data screening is necessary in the case of regional spatial averages.

The suggested method of generalized Haar-series expansion for climate trend analysis has its own strength and weakness. Actually, it represents a compromise between the commonly used analytical approaches and the non-parameteric filtering methods. Its effective feature is that the emphasis is made on the detection of changes in the observational series rather than to find or a priori postulate the actual form of the trend function.

One of the principal problems with the window technique is its sensitivity to changes in the time period of the observations. It is assumed that further development of this method can lead to more flexible choise of the subperiods for which the local mean values are contrasted.

\section{References}

Agee EM (1982) A diagnosis of twentieth century temperature records at West Lafayette, Indiana. Climatic Change 4:399-418

Angell JK (1988) Variations and trends in tropospheric and stratospheric global temperatures 1958-87. J Climate 1:12961313

Callendar GS (1961) Temperature fluctuations and trends over the earth. Quart J Roy Meteor Soc 87:1-2

Dixon KW, Shulman MD (1984) A statistical evaluation of the abilities of climatic averages. J Climate Appl Meteor 23:15421552

Epstein ES (1982) Detecting climate change. J Appl Meteor 21:1172-1181 
Gruza GV, Kachurina LR, Khvostova RN (1987) Choice of a period for estimating the multiyear statistics of the mean monthly surface temperature (in Russian). Meteor Gidrol No $11: 66-74$

Hansen J, Lebedeff S (1987) Global trends of measured surface air temperature. J Geophys Res 92:13345-13372

Hansen J, Lebedeff S (1988) Global surface air temperatures: update through 1987. Geophys Res Lett 15:323-326

Jones PD, Raper SCB, Bradley RS, Diaz HF, Kelley PM, Wigley TML (1986) Northern Hemisphere surface air temperature variations: 1851-1984. J Climate Appl Meteor 25:161-179

Jones PD, Kelly PM (1983) The spatial and temporal characteristics of Northern Hemisphere surface air temperature variations. J Climatol 3:243-252

Jones PD, Wigley TML, Wright PB (1990) Global and hemispheric annual temperature variations between 1881 and 1988 . NDP-022/R1, Carbon Dioxide Information Analysis Center, Oak Ridge National Laboratory, Oak Ridge, Tennessee, USA

Karl TR, Riebsame WE (1984) Identification of 10- to 20-year temperature and precipitation fluctuations in the contiguous United States. J Appl Meteor 23:950-966

Karl TR, Quayle RG (1988) Climate change in fact and in theory: are we collecting facts? Climatic Change 13:5-17

Kim JW, Chang JT, Baker NL, Wilks DS, Gates WL (1984) The statistical problem of climate conversion: determination of the relationship between local and large-scale climate. Mon Wea Rev 112:2069-2077

Knox JL, Higuchi K, Shabbar A, Sargent NE (1988) Secular var- iation of Northern Hemisphere $50 \mathrm{kPa}$ geopotential height. J Climate 1:500-510

Kolmogorov AN, Fomin SzV (1960) Elements of theory of functions and functional analysis (in Russian). Nauka, Moscow

Madden RA, Ramanathan V (1980) Detecting climate change due to increasing carbon dioxide. Science 209:763-768

Riesz F, Sz.-Nagy B (1953) Leçons d'analyse functionelle. 2. édition. Hungarian Academy of Sciences, Budapest

Solow AR (1987) Testing for climatic change: an application of the two-phase regression model. J Climate Appl Meteor 26:1401-1405

Vinnikov KY, Groisman PY (1981) The empirical analysis of $\mathrm{CO}_{2}$ influence on modern changes of the mean annual Northern Hemisphere surface air temperature. Meteor Gidrol 11:30-43

Vinnikov KY, Groisman PY, Lugina KM, Golubev AA (1987) Variatons in Northern Hemisphere mean surface air temperature over 1841-1985. Meteor Gidrol 17:45-55

Wigley TML, Jones PD (1982) Signal-to-noise ratios for surface air temperature and detection of $\mathrm{CO}_{2}$-induced climatic change. In: Moses H, MacCracken MC (eds) Proceedings of the Workshop on First Detection of Carbon Dioxide Effects. DOE Report CONF-8 106214, pp 143-158

Wigley TML, Jones PD, Kelly PM (1986) Empirical climate studies. In: The greenhouse effect, climatic change, and ecosystems. SCOPE 29, pp 271-322

Williams J, van Loon H (1976) The connection between trends of mean temperature and circulation at the surface. Part III: Spring and Autumn. Mon Wea Rev 104:1591-1596 